\title{
Numerical Simulations of Three-Dimensional Drop Collisions
}

\author{
M. R. H. Nobari* \\ University of Michigan, Ann Arbor, Michigan 48109-2121 \\ and \\ Grétar Tryggvason ${ }^{\dagger}$ \\ NASA Lewis Research Center, Cleveland, Ohio 44135
}

\begin{abstract}
Three-dimensional simulations of the off-axis collisions of two drops are presented. The full Navier-Stokes equations are solved by a front-tracking finite difference method that allows a fully deformable fluid interface and the inclusion of surface tension. Depending on whether the interface between the drops is ruptured or not, the drops either bounce or coalesce. For drops that coalesce, the impact parameter, which measures how far the drops are off the symmetry line, determines the eventual outcome of the collision. For small impact parameters the drops coalesce permanently, but for larger impact parameters a grazing collision, where the drops coalesce and then stretch apart again, is observed. The numerically found boundary between coalescing and grazing collisions is compared with experimental observations.
\end{abstract}

\section{Introduction}

$\mathbf{T}$ HE dynamics of fluid drops is of considerable importance in a number of engineering applications and natural processes. The combustion of fuel sprays, spray painting, various coating processes, and rain are only a few of the more common examples. While it is usually the collective behavior of many drops that is of interest, often it is the motion of individual drops that determines the large-scale properties of the system. Thus, for example, the total surface area of drops in sprays depends on the size of the individual drops as well as their number density. Computational models for engineering predictions of spray combustion generally do not resolve the motion of individual drops and must rely on subgrid models where the average effects of the unresolved scales are incorporated into the equations used to predict the large-scale behavior. Many spray models (see Heywood ${ }^{1}$ for a general discussion and references, and Amsden et al. ${ }^{2}$ for a specific example) use point particles to represent the drops. The drop motion is related to the fluid flow by empirical laws for drag, heat transfer, and combustion. Often these laws can be constructed by examining the dynamics of a single drop and how it interacts with the surrounding flow. When the number of drops per unit volume is high, however, it is necessary to allow for the interactions between the drops and their collective effect on the flow. To account for drop collisions, models must contain collision rules that determine whether the drops coalesce or not. ${ }^{2}$ These rules are usually based on experimental investigations of binary collisions of drops, but the small spatial and temporal scales make detailed experimental measurements difficult, and usually the record consists of little more than photographs or a video tape. Since the collision process generally involves large-drop deformation and rupture of the interface separating the drops, it has not been amenable to detailed theoretical analysis. Previous studies are therefore mostly experimental, but sometimes supplemented by greatly simplified theoretical argument.

Two recent experimental investigations of drop collisions can be found in Ashgriz and $\mathrm{Poo}^{3}$ and Jiang et al., ${ }^{4}$ who show several photographs of the various collision modes for both water and hydrocarbon drops. These and other experimental investigations have provided considerable information, and in particular it is now

Presented as Paper 94-0835 at the AIAA 32nd Aerospace Sciences Meeting, Reno, NV, Jan. 10-13, 1994; received Feb. 28, 1995; revision received Sept. 29, 1995; accepted for publication Dec. 3, 1995. Copyright (C) 1996 by the American Institute of Aeronautics and Astronautics, Inc. All rights reserved.

${ }^{*}$ Graduate Student, Department of Mechanical Engineering.

${ }^{\dagger}$ Visiting Researcher, Institute for Computational Mechanics in Propulsion; also Associate Professor, Department of Mechanical Engineering, University of Michigan, Ann Arbor, MI 48109. understood that the outcome of a collision can be classified into five main categories. For head-on collisions there are four main categories: bouncing collision, where the drops collide and separate, retaining their identity; coalescence collision, where two drops become one; separation collision, where the drops temporarily become one but then break up again; and shattering collision, where the impact is so strong that the drops break up into several smaller drops. For off-axis collisions, where the drops approach each other along parallel but separated lines, a fifth category appears, namely, grazing or stretching collisions. Here, the drops coalesce upon contact, but are sufficiently far apart so that they continue along the original path and separate again, sometimes breaking up into more than two drops. The collision type depends on the size of the drops, their relative velocities, their off-axis position, and the physical properties of the fluids involved. For a given drop and ambient fluids some of these collision regimes are not observed. Water drops, for example, usually do not show bouncing at atmospheric pressures, but Qian and $\mathrm{Law}^{5}$ have recently shown experimentally that the film between colliding drops takes longer to drain at higher pressures (and denser ambient fluid), and for sufficiently high pressures bouncing is observed. Other investigations of drop collisions may be found in Bradley and Stow ${ }^{6}$ and Podvysotsky and Shraiber, ${ }^{7}$ for example. The major goal of these investigations has been to clarify the boundaries between the major collision categories and explain how they depend on the parameters of the problem. Simple models used to rationalize experimental findings have been presented by Park and Blair, ${ }^{8}$ Ryley and Bennett-Cowell, ${ }^{9}$ Brazier-Smith et al., ${ }^{10}$ Ashgriz and Poo, ${ }^{3}$ and Jiang et al. ${ }^{4}$

In principle, numerical solutions of the Navier-Stokes equations, where all scales of motion are fully resolved, can provide information not accessible by experimental measurements, but various numerical difficulties associated with moving boundaries between two fluids have made detailed simulations difficult in the past. Nevertheless, several authors have computed the axisymmetric head-on collision of drops with a wall. The first to do so was Foote, ${ }^{11}$ who followed the evolution of rebounding axisymmetric drops at low Weber number using the MAC method. Some of the most recent computations can be found in Fukai et al., ${ }^{12,13}$ who use a moving finite element method. A review of experimental and analytical investigations of collisions of drops with a solid surface has recently been compiled by Rein. ${ }^{14}$ Computations of the head-on collision of two axisymmetric drops have been done by Nobari et al. ${ }^{15}$ who examined the boundary between coalescing and reflecting collision for equal-size drops. Here, we present numerical simulations of three-dimensional, off-axis collisions, where the full Navier-Stokes equations are solved using a front-tracking finite difference method. Another effort to simulate fully three-dimensional drop collisions 
can be found in Lafaurie et al., ${ }^{16}$ who use a method similar to the one used here but without the explicit tracking of the drop surface.

\section{Formulation and Numerical Method}

The numerical technique used for the simulations presented in this paper is the front-tracking/finite difference method of Unverdi and Tryggvason. ${ }^{17,18}$ Since the procedure has been described in detail before, we only outline it briefly here.

The physical problem and the computational domain are sketched in Fig. 1. The domain is a rectangular box, and the drops are initially placed near each end of the domain. A force that is turned off before the drops collide is applied to drive them together. In many cases, the density and viscosity of the ambient fluid are much smaller than that of the drop fluid and thus have only a small effect on the results. Although it may therefore be sufficient to solve only for the fluid motion inside the drop, here we solve for the motion everywhere, both inside and outside the drops. The Navier-Stokes equations are valid for both fluids, and a single set of equations can be written for the whole domain as long as the jump in viscosity and density is correctly accounted for and surface tension is included:

$$
\begin{aligned}
& \frac{\partial \rho \boldsymbol{u}}{\partial t}+\nabla \cdot \rho \boldsymbol{u} \boldsymbol{u}=-\nabla p+\boldsymbol{f}_{\boldsymbol{x}}+\nabla \cdot \boldsymbol{\mu}\left(\nabla \boldsymbol{u}+\nabla \boldsymbol{u}^{T}\right) \\
& -\sigma \int_{S} \kappa \boldsymbol{n} \delta\left(\boldsymbol{x}-\boldsymbol{x}_{f}\right) \mathrm{d} a
\end{aligned}
$$

Here, $u$ is the velocity, $p$ is the pressure, and $\rho$ and $\mu$ are the discontinuous density and viscosity fields, respectively. Surface tension forces are added as a delta force where the interface between the drop and the ambient fluid is. The integral is over the surface of the drop, and $\delta$ is a three-dimensional delta function. The scalar $\sigma$ is the surface tension coefficient, $k$ is twice the mean curvature, and $n$ is an outward-pointing normal to the surface of the drop. The vector $f_{x}$ is a body force used to give the drops their initial velocity. Notice that this form implicitly satisfies the correct stress boundary conditions at the surface of the drop. The above equations are supplemented by the incompressibility condition

$$
\nabla \cdot \boldsymbol{u}=0
$$

which, when combined with the momentum equations, leads to a nonseparable elliptic equation for the pressure. We also have equations of state for the density and viscosity:

$$
\begin{aligned}
& \frac{\partial \rho}{\partial t}+\boldsymbol{u} \cdot \nabla \rho=0 \\
& \frac{\partial \mu}{\partial t}+\boldsymbol{u} \cdot \nabla \mu=0
\end{aligned}
$$

These last two equations simply state that the density and viscosity within each fluid remain constant. In the front-tracking code these equations are solved indirectly by reconstructing the density and viscosity fields from the location of the front at every time step.

Nondimensionalization gives a Weber and a Reynolds number defined by

$$
W e=\frac{\rho_{d} D U^{2}}{\sigma}, \quad R e=\frac{\rho_{d} U D}{\mu_{d}}
$$

where $D$ is the initial diameter of each drop and $U$ is the relative velocity of the drops at impact, defined as the instant when the centers of the drops are one diameter apart. In addition, the density

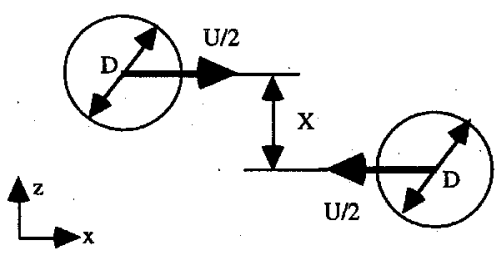

Fig. 1 Computational domain and initial conditions. The drops are initially 2.5 diam apart. The $y$ dimension of the computational box is the same as the $z$ dimension. ratio $r=\rho_{d} / \rho_{o}$ and the viscosity ratio $\lambda=\mu_{d} / \mu_{o}$ must be specified. Here, the subscript $d$ denotes the fluid in the drop, and $o$ the ambient fluid. In off-axis collisions, the drops approach each other along parallel lines that are some distance apart. If this distance is greater than the drop diameter $D$, the drops never touch and no collision takes place: If this distance is zero, we have a head-on collision. To describe off-center collision, a new nondimensional parameter, usually called the impact parameter, is required in addition to the Weber and the Reynolds number defined earlier. This parameter is usually defined as

$$
I=\chi / D
$$

where $\chi$ is the perpendicular distance between the lines that the drops move along before collision. When we present our results, time will be nondimensionalized by $D / U$.

To solve the Navier-Stokes equations we use a fixed, regular, staggered grid and discretize the momentum equations using a conservative, second-order centered finite difference scheme for the spatial variables and an explicit second-order time integration method. The pressure equation; which is nonseparable on account of the difference in density between the drops and the ambient fluid, is solved by a black-and-red successive overrelaxation scheme. Other versions of our code use a multigrid iteration scheme. The novelty of the scheme is the way the boundary, or front, between the drops and the ambient fluid is tracked. The front is represented by separate computational points that are moved by interpolating their velocity from the grid. These points are connected by triangular elements to form a front that is used to keep the density and viscosity stratification sharp and to calculate surface tension. At each time step information must be passed between the front and the stationary grid. This is done by a method similar to the one discussed by Unverdi and Tryggvason, ${ }^{17}$ which spreads the density jump to the grid points next to the front and generates a smooth density field that changes from one density to the other over two to three grid spaces. Although this replaces the sharp interface by a slightly smoother grid interface, it has the advantage that all numerical diffusion is eliminated, since the grid field is reconstructed at each step.

The surface forces are computed from the geometry of the interface and distributed to the grid in the same manner as the density jump. Generally, curvature is very sensitive to minor irregularity in the interface shape, and it is difficult to achieve accuracy and robustness at the same time. However, by computing the surface forces on each surface element by

$$
\sigma \oint_{\Delta} t \times n \mathrm{~d} s
$$

where the integration is over the boundary curve of each element, we ensure that the net surface force on each drop is zero:

$$
\int \sigma \kappa \boldsymbol{n} \mathrm{d} s=\mathbf{0}
$$

where the integration is over the entire drop. Here, $\boldsymbol{n}$ is the outward unit normal and $t$ a unit tangent vector to the boundary curve for each element. This is important for long-time simulations, since even small errors can lead to a net force that moves the drop in an unphysical way.

As the drops move and deform, it is necessary to add and delete points at the front, and to modify the connectivity of the points to keep the front elements of approximately equal size and as well shaped as possible. This is described in Unverdi and Tryggvason. ${ }^{17}$ When the drops are close, we rupture the interface, in several of our computations, by removing surface elements that are very close and nearly parallel, and reconnecting the remaining ones to form a single surface. Here, this restructuring of the interface is done at prescribed time if the interfaces are close enough. While this is rather arbitrary (and we have simply selected a time when the drops look close enough), it allows some control over the dynamics of the rupture, as compared with numerical methods where the front is not tracked and the film would always rupture once it is thinner than a few grid spaces. For a more detailed discussion of this point, see Nobari et al., ${ }^{15}$ where the effect of changing the time of rupture was investigated for head-on collisions. When the governing parameters 
put the collision close to the boundary separating different collision modes, it was found that changing the rupture time could change the outcome of a collision, because of the loss of surface energy when a part of the interface was removed. Away from the boundary, however, the exact time of rupture did not have much effect on the subsequent evolution.

To set the drops in motion, we have several alternatives. If the ambient fluid were absent, the simplest approach would be to assign uniform velocity to the fluid in the drops. However, when the ambient fluid is included, its velocity must be adjusted in such a way that the velocity field is divergence-free. It is possible to violate the divergence-free conditions in the initial conditions (by giving the drops uniform velocity and keeping the ambient fluid at rest, for example), but this will lead to regions of extreme pressure values during the first time step. Another alternative is to use potential flow around a sphere as initial conditions. This is, however, only straightforward for the simplest drop configurations and will furthermore result in a discontinuous velocity across the drop surface and a large shear initially. We have therefore taken a slightly simpler approach and used a force field to accelerate the drops until they reach the desired speed. In addition to being a realistic way of initiating the motion, this is particularly simple to implement in our code. The force is taken as

$$
f_{x}=C\left(\rho-\rho_{o}\right)\left(x-x_{c}\right)
$$

so the force acts only on the drops. Here $C$ is an adjustable constant and $x_{c}$ is midway between the drops. This force is turned off before the actual collision takes place. Initially, the drops are placed with their centers 2.5 diam apart, and $C$ is varied to give different collision velocities.

The method and the codde has been tested in various ways, such as by extensive grid refinement studies, comparison with other published work, and analytical solutions (see also next section). It has also been used to investigate a number of other multifluid problems. In addition to the computations of head-on collisions of drops by Nobari et al., ${ }^{15}$ Unverdi and Tryggvason ${ }^{18}$ simulated the collision of fully three-dimensional bubbles, Ervin ${ }^{19}$ investigated the lift of deformable bubbles rising in a shear flow (see also Esmaeeli et al. ${ }^{20}$ ), Jan and Tryggvason ${ }^{21}$ examined the effect of contaminants on the rise of buoyant bubbles, and Nobari and Tryggvason ${ }^{22}$ followed the coalescence of drops of different sizes. Nas and Tryggvason ${ }^{23}$ presented a simulation of thermal migration of many two-dimensional bubbles.

\section{Results and Discussion}

For the computations presented here, $W e=23, R e=68, r=40$, and $\lambda=20$, but the impact parameter $I$ is varied. For the computations presented in Figs. 5-9 the computational domain is a rectangular box of dimensions $1 \times 1 \times 2$ and is resolved by a $32 \times 32 \times 64$ cubic mesh. The diameter of the drops is 0.4 times the short dimension of the box.

Whereas we have done extensive checks of the accuracy of the axisymmetric code used in Nobari and Tryggvason, ${ }^{22}$ the threedimensional code has not been tested as thoroughly. We have therefore conducted a few calculations of head-on collisions where the results from the three-dimensional simulations can be compared with the axisymmetric results. Figure 2 shows this comparison. The axisymmetric results are to the left, and the fully three-dimensional results to the right. The axisymmetric results have been given a fully three-dimensional appearance by rotating the surface around the symmetry axis. The initial conditions are shown at the top of each column, and the drops are then shown below at equispaced times. The force that acts on the drops initially is turned off before impact (just before the second frame). As the drops collide, they become flatter, and the ambient fluid between them is pushed away, leaving a thin film of fluid between the drops. Here, this film is not removed and the drops therefore rebound, recovering their spherical shape. Obviously, the results are in good agreement. Figure 3 shows a more quantitative comparison, where we plot the $x$ position of the center of mass for the drops in Fig. 2, as well as for drops computed on a coarser grid $(16 \times 16 \times 32)$. Time is nondimensionalized by $D / U$, and the origin is set at the time when the forces are turned off. The

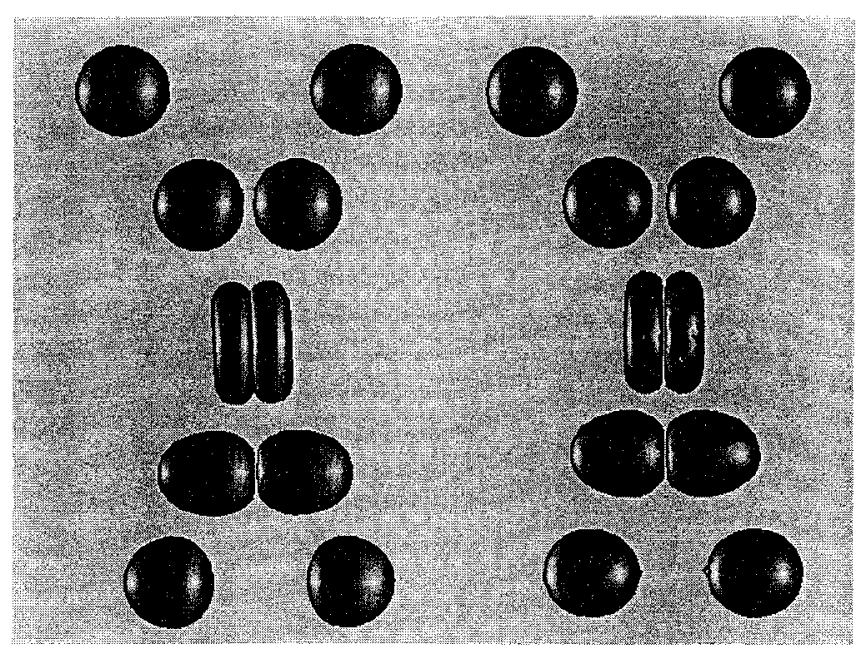

Fig. 2 Comparison between a fully three-dimensional simulation (right) and results obtained by an axisymmetric code (left). The initial conditions are shown at the top of each column and the solution is then shown at three equispaced times for each run.

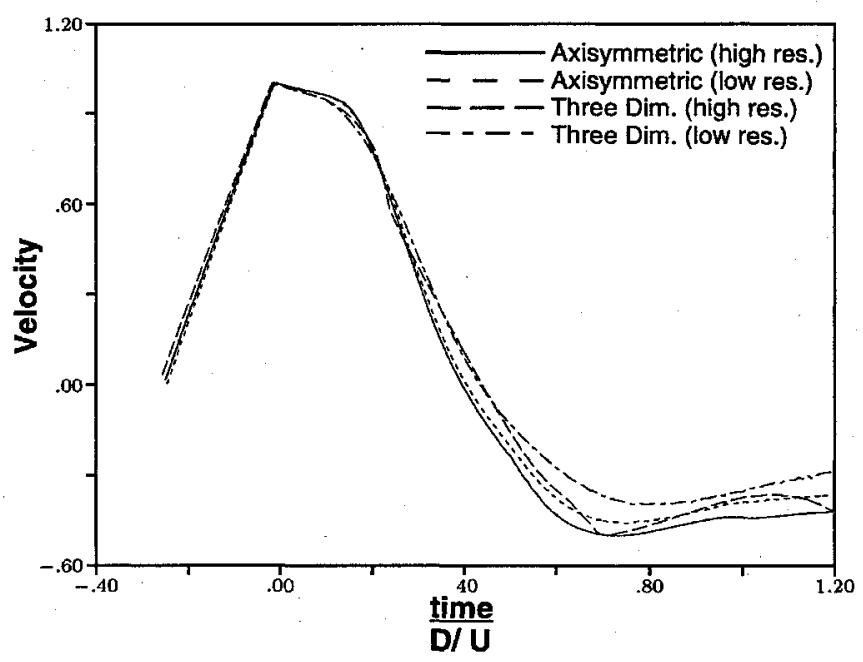

Fig. 3 Relative velocity $U$ of the center of mass of one drop vs time, as computed by both the fully three-dimensional code and an axisymmetric one, for two different resolutions.

agreement is reasonably good, although the coarse-grid results are not in as good agreement with each other as the finer-grid results are.

Another grid resolution study is presented in Fig. 4. Since a finegrid three-dimensional computations would be expensive, we have used a two-dimensional version of the code here. Except for the dimensionality, the codes are identical. The simulations have been done on a $16 \times 32$ grid, $32 \times 64$ grid, and $64 \times 128$ grid. The middle resolution corresponds to what is used for the three-dimensional simulations in Figs. 5-9. In Fig. 4 the surfaces of the drops are plotted at three times for the two finer resolutions in the top three frames. The top frame is at the time when the force driving the drops together is turned off, in the second frame the drops have collided, and the third frame shows the drops starting to rebound. In the bottom frame the results for all three resolutions, at the third time, are plotted. The results for the two finer grids differ only by a small amount, and even though the coarse-grid results are obviously not fully converged, the bottom frame shows that overall evolution is well reproduced. The behavior seen here is fairly typical of the method described above: very coarse resolution gives qualitatively correct results that converge rapidly under grid refinement.

In Fig. 5, the off-axis collision of two drops for $I=0.75$ is shown. The pair is shown at several equispaced times, beginning with the initial position at the top of the figure. Unlike in Fig. 3, here we put $t=0.0$ when the distance between the centers of the drops is 1 diam. If the drops remained completely spherical, this would be the time when they first touched. Once the drops have the desired 

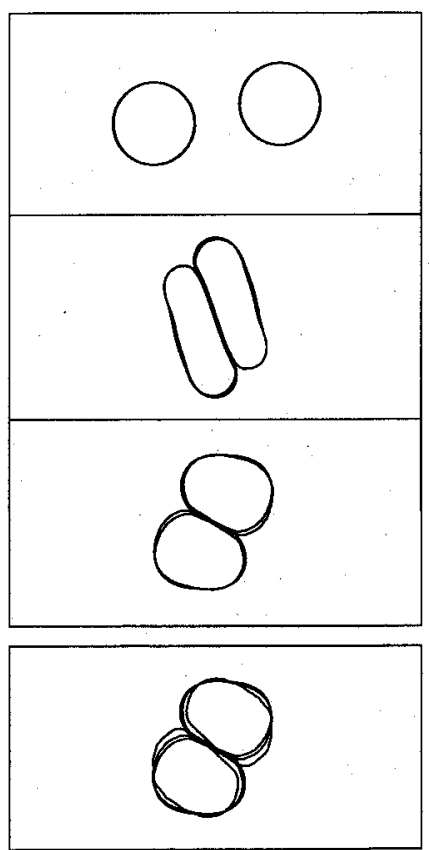

Fig. 4 Two-dimensional grid resolution study. The drop surface computed on a $16 \times 32$, a $32 \times 64$, and a $64 \times 128$ grid. Results from the two finer grids, at three times, are shown in the top three frames, and results from all three grids at the last time are shown in the bottom frame.

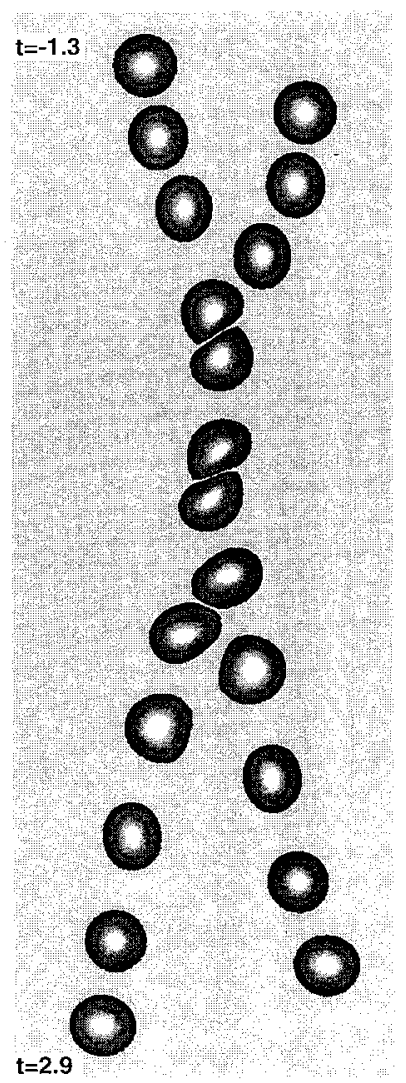

Fig. 5 Bouncing collision. Here $I=0.75$ and the drops are not allowed to coalesce. The initial conditions are shown at the top, and the drops are then shown every 0.42 time unit.

velocity, around the third frame from the top, the force that is applied to drive the drops together is turned off. The drops continue to move together, and in the fourth frame they have collided, deforming as they do so. Since the collision parameter is large, the drops slide past each other and continue along their original paths. The bottom four frames show the motion of the drops after the collision. During the collision the drops become nearly flat where they face each other, and as the drops slide past each other the fluid layer between them
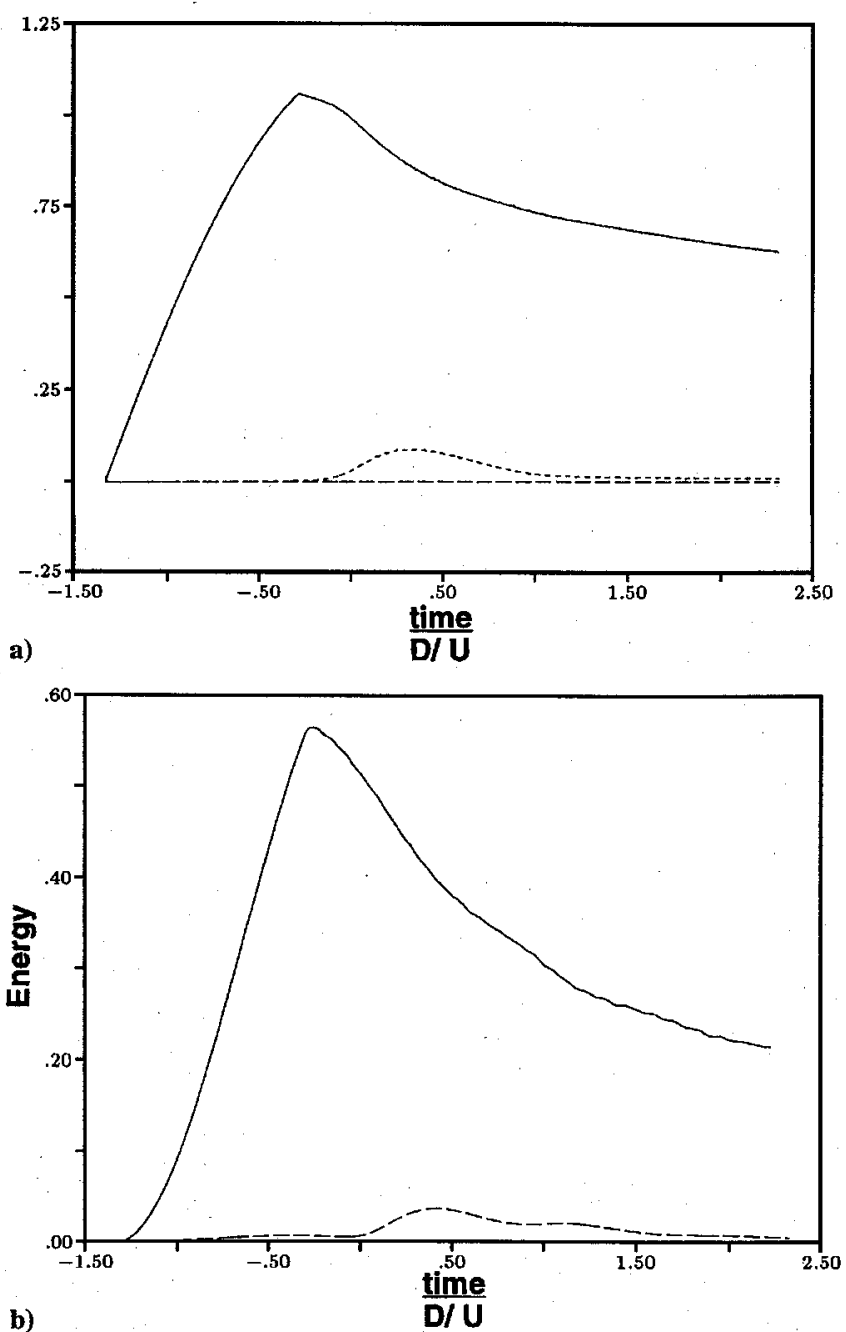

Fig. 6 a) Center-of-mass velocity of one drop from the computation in Fig. $5(-, U$; ---, V; and ..., W) and b) kinetic $(-)$ and surface tension (--) energy of one drop.

becomes progressively thinner. If it becomes thin enough it should rupture, but here we have not allowed that to happen (as seen in Fig. 7, rupture of this film will change the resulting evolution considerably). In Fig. 6, the velocity components of the center of mass of one of the drops (Fig. 6a) and the kinetic and the surface tension energy (Fig. 6b) are plotted vs time. The solid curve in Fig. $6 \mathrm{a}$ is the velocity in the horizontal direction. It increases as the force accelerates the drops together, and then decreases slightly because of the drag from the outer fluid after the force is turned off. When the drops actually collide, it decreases more rapidly, but eventually resumes a nearly constant decay rate after the collision is over. The velocity component in the vertical direction (short dashes) is nonzero only during the actual collision, suggesting that the vertical movement of the drop is more a result of deformation than a change of path. The kinetic energy in Fig. $6 \mathrm{~b}$ shows similar behavior to the velocity: it decreases slowly after the force is turned off, decreases more rapidly during collision, and then resumes slow decay. The surface tension energy rises during the collision as the drop deforms, thus contributing to the reduction in the kinetic energy. Notice that the drop oscillates slightly after the collision, as seen in the surface tensión energy plot.

Although bouncing is observed for real drops, it is actually a rather rare outcome of a collision, only seen when the drops deform and trap the ambient fluid between them and the drop velocity is sufficiently large so the film does not have time to drain before the drops rebound. To investigate the behavior of drops that coalesce, we have written software to automatically remove the front bounding the thin film between the drops at a prescribed time and allow the drops to coalesce. Figure 7 shows the results of two computations where the drops coalesce. All parameters are the same as in Fig. 2, 

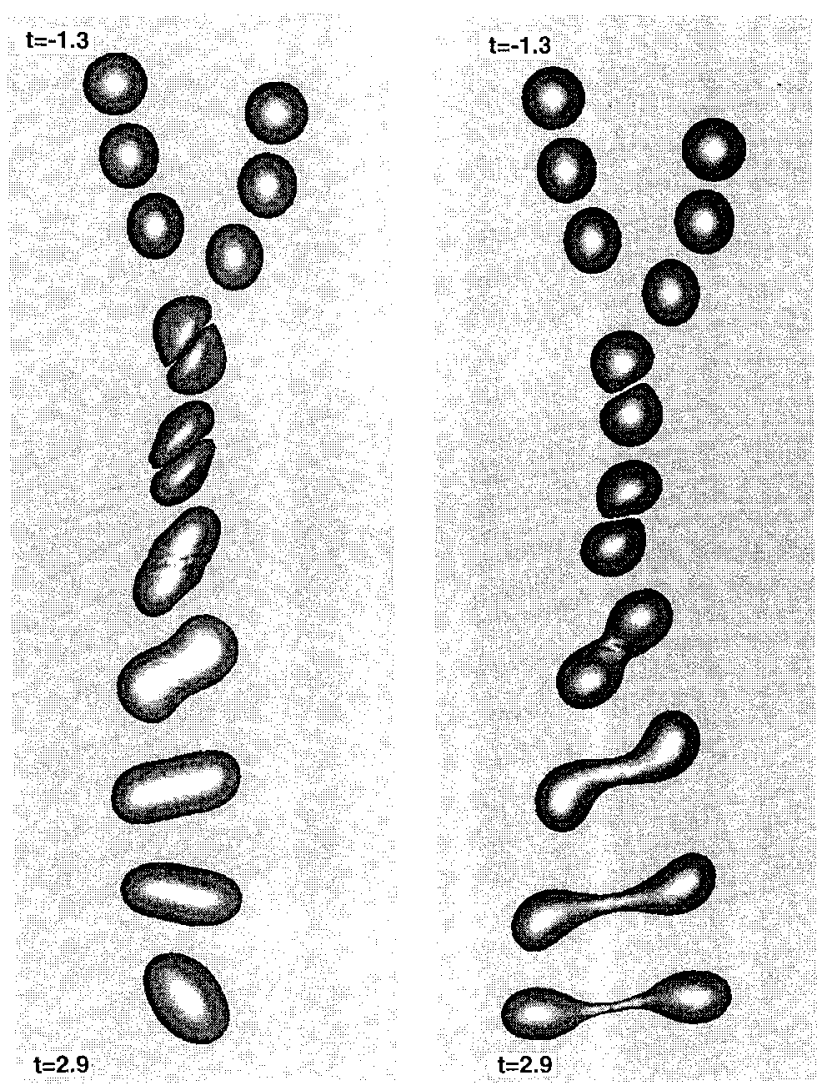

Fig. 7 Coalescing collisions. The initial conditions are shown at the top of the figure, and the pair is then shown every 0.42 time units. The film is ruptured at $t=0.46$ in both cases, but the impact parameter is different for the two runs. In the left column $I=0.5$, and in the right column $I=$ 0.825. For the small impact parameter, the drops coalesce permanently, but for the larger one they separate again.

except that in the left column $I=0.50$, and in the right column $I=0.825$. The film between the drops is ruptured at time 0.46 for both runs. For the small-impact-parameter case, the drops deform considerably during the initial impact, as observed for head-on collisions, but the impact parameter is sufficiently large so the drops still slide past each other. As the film is ruptured and the drops coalesce, the momentum of each drop is sufficiently large so the large combined drop continues to elongate. Eventually, however, surface tension overcomes the stretching and the drop is pulled into a spherical shape. Because of the velocity of the drops that coalesced, the combined drop rotates. Whereas the small-impact-parameter drops are in many way similar to drops undergoing a head-on collision, the large-impact-parameter drops in Fig. 7b deform only slightly as they collide. When the interface between them is ruptured, they have nearly passed each other, and after rupture their momentum is sufficiently large so they continue along their original path and stretch the fluid column connecting them until it is near breaking. We have not written the software necessary for rupturing the filament connecting the drops and therefore must stop the computations at this point. Notice that the coalesced drop rotates, as the small-impact-parameter one did, although much less. Although we have not attempted detailed quantitative comparisons, the coalescing collision in Fig. $6 \mathrm{a}$ and the grazing collision in Fig. $6 \mathrm{~b}$ are in good qualitative agreement with the experimental photographs in Ashgriz and Poo, ${ }^{3}$ Jiang et al., ${ }^{4}$ and Qian and Law, ${ }^{5}$ for example.

In Fig. 8, the surface tension energy, the kinetic energy, and the total energy of the drops from Fig. 7 are plotted vs time. Initially, the kinetic energy is increased by the force that accelerates the drops together. After the force has been turned off, the drops move a short distance before colliding. Since the ambient fluid has a nonzero viscosity, kinetic energy is dissipated by friction and the drops slow down. As the drops come in contact, the kinetic energy of the small-impact-parameter drops decreases rapidly, but the largeimpact-parameter drops are not affected to any significant degree. Similarly, the surface tension energy of the small-impact-parameter
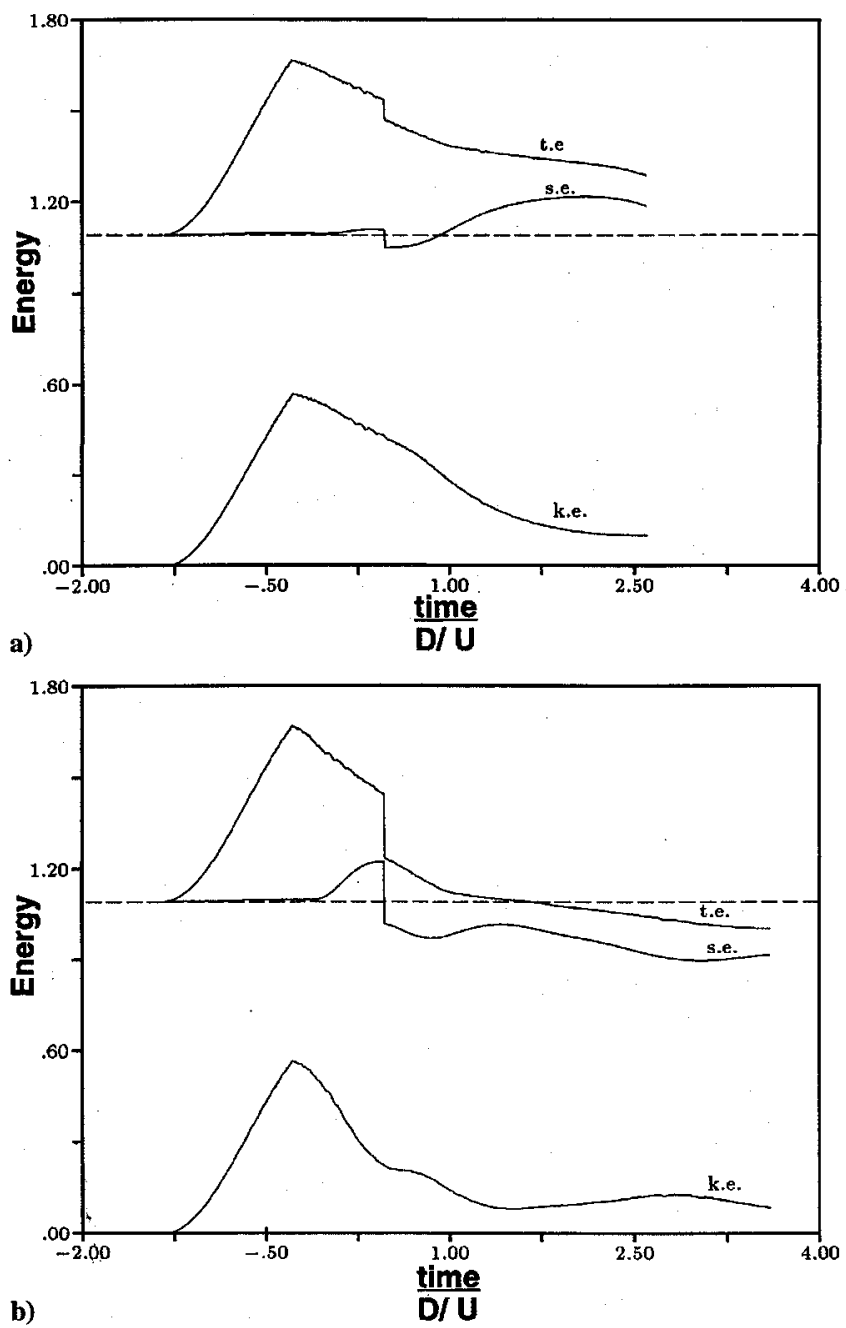

Fig. 8 Energies for the drops in Fig. 6: a) $I=0.5$ and b) $I=0.825$. The total energy, the surface energy, and the kinetic energy of the drops are plotted vs time.

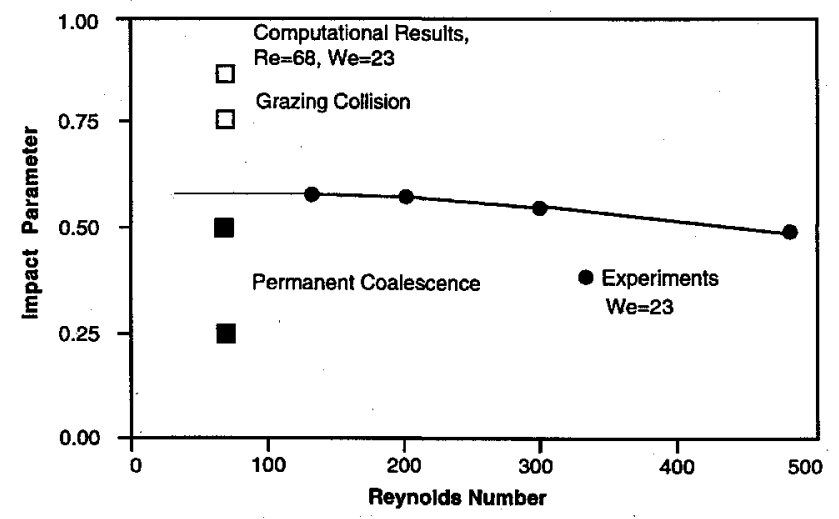

Fig. 9 Boundaries between coalescing and grazing collisions in the $R e-I$ plane for $W e=23$. The solid circles, connected by a line, are experimental data from Jiang et al. ${ }^{4}$ The squares are computed results.

drops increases as the drops deform, but the surface tension energy of the other drops hardly increases at all, since the drops remain almost spherical. When the film between the drops is ruptured, part of the drops' surface is removed and the surface energy reduced. This reduction is larger for the small-impact-parameter drops, since the area removed is larger. Initially, the kinetic energy of the large-impact-parameter drops is nearly unaffected (and continues to be dissipated at the same rate as before the drops collide), but as the coalesced drop starts to stretch and the surface tension energy to increase, the kinetic energy decreases sharply. As the filament between the drops starts to neck down, the increase in surface 
area stops and the kinetic energy levels off. For the small-impactparameter drops, the rupture takes place near the time of maximum deformation, and surface energy is initially converted into kinetic energy as the drop adjusts to the new shape. The momentum of the drop before impact is, however, sufficiently large so the drop is elongated as the fluid of the original drops continue along the paths they were following before collision. This leads to an increase in surface tension energy and a decrease in kinetic energy. When the surface tension energy reaches maximum, the kinetic energy is not zero, because of the rotational motion of the coalesced drop.

For modeling of droplet collisions, the major question is whether the collision results in a single drop or not. Figure 9 shows our computations in the $I-R e$ plane. In addition to the computations shown in Fig. 6, we have conducted two other calculations at different impact parameters. The runs that lead to a coalesced drop are shown by black squares, and those leading to grazing collision as open squares. We have also plotted the experimental results of Jiang et al. ${ }^{4}$ for the boundary between these two collision modes for $W e=23$. Their results do not extend down to the Reynolds number simulated here, but since the boundary is only weakly dependent on the Reynolds number, it seems safe to extrapolate their results to our Reynolds number. The thin line shows this extrapolation, showing that the numerical results are consistent with the experiments.

\section{Conclusion}

In this paper we present numerical predictions of fully threedimensional off-axis collisions of two drops. These computations, which require about $10 \mathrm{~h}$ on a Cray X-MP, are done on a coarse mesh and are therefore limited to small Reynolds and Weber numbers. Nevertheless, they do demonstrate well the feasibility of such predictions. In Ref. 15 we showed simulations of head-on collisions at both higher $W e$ and higher $R e$. There we took advantage of the axisymmetry of the problem and could afford a much finer grid. We believe that comparable three-dimensional simulations are simply a matter of finer resolution, using either a larger number of grid points or adaptive gridding.

The principle weakness of the simulations presented here is the ad hoc way the film between the drops is ruptured for coalescing drops. We have prescribed the time of rupture, but it is a simple matter to use other criteria such as the thickness of the film. If we used a numerical method that did not explicitly track the interface, ${ }^{16}$ the drops would always coalesce once they were close enough. However, in reality the rupture should reflect the time it takes for the film to drain down to dimensions where it is unstable to attractive interface forces. Since resolving the flow in the film completely is likely to require excessively fine grids (even using an adaptive gridding strategy), we believe that the most promising approach would be to couple a simulation like ours with an analytical model of the film draining. Such models have been developed by a number of authors, generally using very simplified models for the drop evolution. For a recent model and other references, see Jacqmin and Foster. ${ }^{24}$ Since our method explicitly tracks the surface of the drop, the incorporation of such a subgrid model for the film should be possible.

\section{Acknowledgments}

Part of this work was done while one of the authors (G. T.) was visiting the Institute for Computational Mechanics in Propulsion at NASA Lewis Research Center. This work is supported in part by NASA Grant NAG3-1317 and National Science Foundation Grant CTS-913214. Some of the computations. were done at the San Diego Supercomputing Center, which is funded by the National Science Foundation. We would like to acknowledge discussions with D. Jacqmin at the NASA Lewis Research Center.

\section{References}

${ }^{1}$ Heywood, J. B., Internal Combustion Engine Fundamentals, McGrawHill, New York, 1988.

${ }^{2}$ Amsden, A. A., O'Rourke, P. J., and Butler, T. D., "KIVA-II: A Computer Program for Chemically Reactive Flows with Sprays," Los Alamos National Lab., Rept. LA-11560-MS, 1989.

${ }^{3}$ Ashgriz, N., and Poo, J. Y., "Coalescence and Separation in Binary Collisions of Liquid Drops," Journal of Fluid Mechanics, Vol. 221, 1990, pp. 183-204.

${ }^{4}$ Jiang, Y. J., Umemura, A., and Law, C. K., "An Experimental Investigation on the Collision Behavior of Hydrocarbon Droplets," Journal of Fluid Mechanics, Vol. 234, 1992, pp. 171-190.

${ }^{5}$ Qian, J., and Law, C. K., "Effects of Ambient Gas Pressure on Droplet Collision," AIAA-94-0681, 1994.

${ }^{6}$ Bradley, S. G., and Stow, C. D., "Collision Between Liquid Drops," Philosophical Transactions of the Royal Society of London, Series A: Mathematical and Physical Sciences, Vol. 287, 1978, pp. 635-678.

${ }^{7}$ Podvysotsky, A. M., and Shraiber, A. A., "Coalescence and Breakup of Drops in Two Phase Flows," International Journal of Multiphase Flow, Vol. 10, No. 2, 1984, pp. 195-209.

${ }^{8}$ Park, J. Y., and Blair, L. M., "The Effect of Coalescence on Drop Size Distribution in an Agitated Liquid-Liquid Dispersion," Chemical Engineering Science, Vol. 30, No. 9, pp. 1057-1064.

${ }^{9}$ Ryley, D. J., and Bennett-Cowell, B. N., "The Collision Behavior of Steam-Borne Water Drops," International Journal of Mechanical Sciences, Vol. 9, No. 12, 1967, pp. 817-833.

${ }^{10}$ Brazier-Smith, P. R., Jennings, S. G., and Latham, J., "The Interaction of Falling Water Drops: Coalescence," Proceedings of the Royal Society of London, Series A, Mathematical and Physical Sciences, Vol. 326, 1972, pp. $393-408$.

${ }^{11}$ Foote, G. B., "The Water Drop Rebound Problem: Dynamics of Collision," Journal of Atmospheric Science, Vol. 32, 1975, pp. 390-402.

${ }^{12}$ Fukai, J., Zhao, Z., Poulikakos, D., Megaridis, C. M., and Miyatake, O., "Modeling of the Deformation of a Liquid Droplet Impinging Upon a Flat Surface," Physics of Fluids A, Vol. 5, No. 11, 1993, pp. 2589-2599.

${ }^{13}$ Fukai, J., Shiiba, Y., Yamamoto, T., Miyatake, O., Poulikakos, D., Megaridis, C. M., and Zhao, Z., "Wetting Effects on the Spreading of a Liquid Droplet Colliding with a Flat Surface: Experiments and Modeling," Physics of Fluids A, Vol. 7, No. 1, 1995, pp. 236-247.

${ }^{14}$ Rein, M., "Phenomena of Liquid Drop Impact on Solid and Liquid Surfaces," Fluid Dynamics Research, Vol. 12, 1993, pp. 61-93.

${ }^{15}$ Nobari, M. R. H., Jan, Y.-J., and Tryggvason, G., "Head-On Collision of Drops-A Numerical Investigation," Physics of Fluids, Vol. 8, No. 1, 1996, pp. 29-42.

${ }^{16}$ Lafaurie, B., Nardone, C., Scardovelli, R., Zaleski, S., and Zanetti, G., "Modelling Merging and Fragmentation in Multiphase Flows with SURFER," Journal of Computational Physics, Vol. 113, No. 1, 1994, pp. 134-147.

${ }^{17}$ Unverdi, S. O., and Tryggvason, G., "A Front Tracking Method for Viscous Incompressible Flows," Journal of Computational Physics, Vol. 100, No. 1, 1992, pp. 25-37.

${ }^{18}$ Unverdi, S. O., and Tryggvason, G., "Multifluid Flows," Physica D, Vol. 60,1992 , pp. $70-83$.

${ }^{19}$ Ervin, E. A., "Full Numerical Simulations of Bubbles and Drops in Shear Flow," Ph.D. Thesis, Dept. of Mechanical Engineering, Univ. of Michigan, Ann Arbor, MI, 1993.

${ }^{20}{ }^{E}$ Esmaeeli, A., Ervin, E. A., and Tryggvason, G., "Numerical Simulations of Rising Bubbles," Bubble Dynamics and Interface Phenomena, edited by J. R. Blake, J. M. Boulton-Stone, and N. H. Thomas, Kluwer Academic, 1994, pp. 247-255.

${ }^{21}$ Jan, Y.-J., and Tryggvason, G., "Computational Studies of Contaminated Bubble," Physics of Fluids (submitted for publication).

${ }^{22}$ Nobari, M. R. H., and Tryggvason, G., "Coalescence of Initially Stationary Drops," Journal of Fluid Mechanics (submitted for publication).

${ }^{23}$ Nas, S., and Tryggvason, G., "Computational Investigation of the Thermal Migration of Bubbles and Drops," Fluid Mechanics Phenomena in Microgravity, edited by D. A. Siginer, R. L. Thompson, and L. M. Trefethen, AMD-Vol. 174/FED-Vol. 175, American Society of Mechanical Engineers, 1993, pp. 71-83.

${ }_{24}$ Jacqmin, D., and Foster, M. R.,"The Evolution of Thin Films Generated by the Collision of Highly Deforming Droplets," Physics of Fluids (submitted for publication). 